2011-0111

\title{
Application of the Slow Invariant Manifold Correction for Diffusion
}

\author{
Joshua D. Mengers* and Joseph M. Powers ${ }^{\dagger}$ \\ University of Notre Dame, Notre Dame, Indiana 46556, USA
}

\begin{abstract}
Slow invariant manifolds (SIM) are calculated for spatially inhomogeneous closed reactive systems to obtain a model reduction. A robust method of constructing a onedimensional SIM by calculating equilibria and then integrating along heteroclinic orbits is extended to two new cases: i) adiabatic systems, and ii) spatially inhomogeneous systems with simple diffusion. The adiabatic condition can be modeled as a new algebraic constraint in the limit of unity Lewis number. Diffusion effects on the SIM are examined for systems with small characteristic lengths. A diffusion correction is obtained by using a Galerkin method to project the infinite dimensional dynamical system onto a low dimensional approximate inertial manifold. This projection rigorously accounts for the coupling of reaction and diffusion processes, and an analytic length and time scale coupling is shown. An example is demonstrated on a system of $N O$ production which highlights the correlation between an established isothermal spatially homogeneous technique and the new techniques.
\end{abstract}

\section{Introduction}

Reactive flow problems are known to display multi-scale phenomena that cause challenges in numerical simulations. Verification requires grid resolution that captures the full range of scales in both space and time. Large disparity in scales induces simulations that require significant computational effort. A disparity in temporal scales can be caused by the reaction mechanism alone; the addition of diffusion introduces additional time scales. Recently, effort has been expended in identification of model reduction techniques for reactive flows in order to reduce the computational cost, while maintaining as much consistency with the underlying reactive flow physics as possible. The reviews of Griffiths ${ }^{1}$ and $\mathrm{Lu}$ and $\mathrm{Law}^{2}$ are useful. Most of the methods described therein address only reaction mechanisms. Some current research that extends these methods to systems with diffusion are Singh et al. ${ }^{3}$ Ren and Pope, ${ }^{4}$ Davis,,${ }^{5,6}$ Bykov and Maas, ${ }^{7}$ Lam, ${ }^{8}$ Adrover et al. ${ }^{9}$ and Goussis et al. ${ }^{10}$

The study of Davis and Skodje ${ }^{11}$ is particularly relevant. In their study, which was performed on spatially homogeneous reactive systems, the authors calculate a one-dimensional slow invariant manifold (SIM) by integrating a heteroclinic orbit between the system's physical and non-physical equilibria. This technique has recently been refined by Al-Khateeb et al. ${ }^{12}$ to examine realistic systems with a larger number of species. The SIM is a unique trajectory of the dynamical system that describes the long time dynamics of the system's evolution efficiently. The SIM has almost exclusively been used for spatially homogeneous isothermal systems.

The present work contains two key extensions in which we examine: i) adiabatic systems, and ii) spatially inhomogeneous systems. We first present the governing partial differential equations (PDEs) for adiabatic systems with diffusion. We then give reductions via a Galerkin projection method for the system of PDEs to reduce it to a small system of non-linear ordinary differential equations (ODEs). In the limit of small length scales these ODEs reduce to the spatially homogeneous problem. For small, finite length scales, we find correction terms to account for diffusion. We then give results for the spatially homogeneous limit of isothermal and adiabatic cases. These are followed by spatially inhomogeneous isothermal results. Each of

\footnotetext{
*Ph.D. Candidate, Department of Aerospace and Mechanical Engineering, University of Notre Dame, AIAA Student Member.

$\dagger$ Professor, Department of Aerospace and Mechanical Engineering, University of Notre Dame, AIAA Associate Fellow.

Copyright (C) 2011 by the American Institute of Aeronautics and Astronautics, Inc. The U.S. Government has a royalty-free license to exercise all rights under the copyright claimed herein for Governmental purposes. All other rights are reserved by the copyright owner.
} 
these are shown for the same Zel'dovich reaction mechanism of $N O$ production studied by Al-Khateeb et al. ${ }^{12}$

\section{Model}

We model a system of detailed reaction kinetics of $N$ species reacting in $J$ reactions with diffusion in one spatial dimension. The key assumptions in our model include an ideal mixture of calorically perfect ideal gases, constant specific heat, negligible advection, constant pressure, a single constant mass diffusivity, and a constant thermal conductivity.

\section{II.A. Balance Laws}

Our governing species evolution equation is

$$
\rho \frac{\partial Y_{i}}{\partial t}+\frac{\partial j_{i}^{m}}{\partial x}=\mathrm{M}_{i} \dot{\omega}_{i}\left(Y_{n}, T\right), \quad \text { for } i, n \in[1, N],
$$

where for $i=1, \ldots, N, Y_{i}, j_{i}^{m}, \dot{\omega}_{i}$, and $\mathrm{M}_{i}$, are mass fraction, diffusive mass flux, molar production rate, and molecular mass of species $i$, respectively; $\rho$ is the density, and $T$ is the temperature. The energy equation is

$$
\rho \frac{\partial h}{\partial t}+\frac{\partial j^{q}}{\partial x}=0
$$

where $h$ is the enthalpy, and $j^{q}$ is the diffusive energy flux. Our model is on a domain of $x \in[0, \ell]$ and $t \in[0, \infty)$. The initial conditions are

$$
Y_{i}(x, t=0)=\tilde{Y}_{i}(x), \quad \text { for } i \in[1, N], \quad \text { and } \quad T(x, t=0)=\tilde{T}(x),
$$

where the ${ }^{\sim}$ denotes the initial state. We select homogeneous Neumann boundary conditions:

$$
\left.\frac{\partial Y_{i}}{\partial x}\right|_{x=0}=\left.\frac{\partial Y_{i}}{\partial x}\right|_{x=\ell}=0, \quad \text { for } i \in[1, N], \quad \text { and }\left.\quad \frac{\partial T}{\partial x}\right|_{x=0}=\left.\frac{\partial T}{\partial x}\right|_{x=\ell}=0
$$

\section{II.B. Constitutive Equations}

To complete the system, a set of constitutive equations is specified. The diffusive fluxes are

$$
\begin{aligned}
j_{i}^{m} & =-\rho \mathcal{D} \frac{\partial Y_{i}}{\partial x}, \\
j^{q} & =-k \frac{\partial T}{\partial x}+\sum_{i=1}^{N} h_{i}^{f} j_{i}^{m},
\end{aligned}
$$

where $\mathcal{D}$ is the constant mass diffusivity, $k$ is the constant thermal conductivity, and $h_{i}^{f}$ is the enthalpy of formation of species $i$. Thus, our homogeneous boundary conditions result in no diffusive mass or energy flux at either boundary. The enthalpy is given by the caloric equation of state,

$$
h=\sum_{i=1}^{N} Y_{i}\left(c_{P i}\left(T-T^{o}\right)+h_{i}^{f}\right),
$$

where $c_{P i}$ is the constant specific heat at constant pressure of species $i$, and $T^{o}=300 \mathrm{~K}$ is the reference temperature. The density is given by the ideal gas equation of state for a mixture which obeys Dalton's law:

$$
\rho=\frac{P_{o}}{\overline{\mathfrak{R}} T} \sum_{i=1}^{N} \frac{\mathrm{M}_{i}}{Y_{i}},
$$

where $P_{o}$ is the constant pressure, and $\overline{\mathfrak{R}}=8.314 \times 10^{7} \mathrm{erg} /($ mol K$)$ is the universal gas constant. 
The reaction source terms are

$$
\begin{aligned}
\dot{\omega}_{i} & =\sum_{j=1}^{J} \nu_{i j} r_{j}, \quad \text { for } i \in[1, N], \\
r_{j} & =k_{j}\left(\prod_{i=1}^{N}\left(\frac{\rho Y_{i}}{\mathrm{M}_{i}}\right)^{\nu_{i j}^{\prime}}-\frac{1}{K_{j}^{c}} \prod_{i=1}^{N}\left(\frac{\rho Y_{i}}{\mathrm{M}_{i}}\right)^{\nu_{i j}^{\prime \prime}}\right), \quad \text { for } j \in[1, J], \\
k_{j} & =a_{j} T^{\beta_{j}} \exp \left(\frac{-\bar{E}_{j}}{\overline{\mathfrak{R} T}}\right), \quad \text { for } j \in[1, J], \\
K_{j}^{c} & =\exp \left(\frac{-\sum_{i=1}^{N} \bar{g}_{i}^{o} \nu_{i j}}{\overline{\mathfrak{R} T}}\right), \quad \text { for } j \in[1, J],
\end{aligned}
$$

where for $j=1, \ldots, J, r_{j}, k_{j}$, and $K_{j}^{c}$ are the reaction rate, Arrhenius rate, and equilibrium constant of reaction $j$, respectively. The reaction-species coefficient matrix, $\nu_{i j} \equiv \nu_{i j}^{\prime \prime}-\nu_{i j}^{\prime}$, is of $\operatorname{dimension} N \times J$ and is segregated into forward, $\nu_{i j}^{\prime}$, and reverse, $\nu_{i j}^{\prime \prime}$, reactions. Eqs. (1-12) form a complete set of equations.

\section{II.C. Model Reduction}

Here we describe a series of reductions of Eqs. (1-12).

\section{II.C.1. Generalized Shvab-Zel'dovich}

We can further simplify the system using reductions similar to those in Lam and Bellan, ${ }^{13}$ who label them as generalized Shvab-Zel'dovich relations. We construct a matrix $\varphi_{l i}$ of dimension $L \times N$, where the $L$ row vectors span the left null space of matrix $\nu_{i j}$ :

$$
\sum_{i=1}^{N} \varphi_{l i} \nu_{i j}=0, \quad \text { for } l \in[1, L], \text { and } j \in[1, J]
$$

Operating on Eq. (9) with $\varphi_{l i}$ and summing on $i$, we get

$$
\sum_{i=1}^{N} \varphi_{l i} \sum_{j=1}^{J} \nu_{i j} r_{j}=\sum_{j=1}^{J} r_{j} \underbrace{\sum_{i=1}^{N} \varphi_{l i} \nu_{i j}}_{=0}, \quad \text { for } l \in[1, L]
$$

therefore

$$
\sum_{i=1}^{N} \varphi_{l i} \dot{\omega}_{i}=0, \quad \text { for } l \in[1, L]
$$

With this simplification, we can apply the operator $\varphi_{l i}$ on Eq. (1), use the diffusive mass flux from Eq. (5), and sum over all species to obtain

$$
\frac{\partial}{\partial t}\left(\sum_{i=1}^{N} \varphi_{l i} \frac{Y_{i}}{\mathrm{M}_{i}}\right)-\mathcal{D} \frac{\partial^{2}}{\partial x^{2}}\left(\sum_{i=1}^{N} \varphi_{l i} \frac{Y_{i}}{\mathrm{M}_{i}}\right)=0, \quad \text { for } l \in[1, L] .
$$

If the initial conditions are spatially homogeneous in the quantity

$$
\sum_{i=1}^{N} \varphi_{l i} \frac{Y_{i}}{\mathrm{M}_{i}}, \quad \text { for } l \in[1, L]
$$

and there are no perturbations from the boundary conditions, then Eq. (16) can be integrated to yield

$$
\sum_{i=1}^{N} \varphi_{l i} \frac{Y_{i}}{\mathrm{M}_{i}}=\sum_{i=1}^{N} \varphi_{l i} \frac{\tilde{Y}_{i}}{\mathrm{M}_{i}}, \quad \text { for } l \in[1, L]
$$


which is a set of $L$ algebraic relations for $Y_{i}$. This suggests that we need not solve for the nonlinear dynamics of all $N$ species, but can focus attention on $N-L$ species and use Eq. (18) to determine the remaining ones. Note that each individual $Y_{i}(x)$ need not be spatially homogeneous, only the values of the constraints defined in Eq. (17). Each row vector of $\varphi_{l i}$ can be correlated to a physical constraint on our system such as the conservation of elements or the conservation of total moles for systems with purely bimolecular reactions.

We can further simplify the system by casting the energy equation in a related Shvab-Zel'dovich form. We assume our system has a constant mixture specific heat,

$$
c_{P}=\sum_{i=1}^{N} Y_{i} c_{P i}
$$

A constant mixture specific heat is typically the result of the assumption of a single temperature-independent constant specific heat for all species; however, in systems with only bimolecular reactions, a constant mixture specific heat can also be realized. We evaluate the limit where the Lewis number is unity,

$$
L e \equiv \frac{k}{\rho c_{P} \mathcal{D}}=1 \text {. }
$$

This demands that mass and energy diffuse at the same rate, which is a reasonable assumption since both are driven by molecular collisions in gas flames.

Evaluating the time derivative of enthalpy from Eq. (7) yields

$$
\rho \frac{\partial h}{\partial t}=\rho c_{P} \frac{\partial T}{\partial t}+\rho \sum_{i=1}^{N} h_{i}^{f} \frac{\partial Y_{i}}{\partial t}
$$

and taking the spatial derivative of the diffusive energy flux term from Eq. (6) gives

$$
\frac{\partial j^{q}}{\partial x}=-k \frac{\partial^{2} T}{\partial x^{2}}-\rho \mathcal{D} \sum_{i=1}^{N} h_{i}^{f} \frac{\partial^{2} Y_{i}}{\partial x^{2}}
$$

We can insert Eqs. (21) and (22) into Eq. (2), cancel like terms, substitute $\mathcal{D}=k /\left(\rho c_{P}\right)$, and collect the spatial and temporal derivatives to reduce the energy equation to a heat equation in enthalpy:

$$
\frac{\partial}{\partial t} \underbrace{\left(c_{P}\left(T-T^{o}\right)+\sum_{i=1}^{N} h_{i}^{f} Y_{i}\right)}_{h}=\mathcal{D} \frac{\partial^{2}}{\partial x^{2}} \underbrace{\left(c_{P}\left(T-T^{o}\right)+\sum_{i=1}^{N} h_{i}^{f} T_{i}\right)}_{h} .
$$

If the enthalpy is initially spatially homogeneous, and there are no perturbations at the boundary, then Eq. (23) can be integrated to form an algebraic relation where enthalpy remains constant in both space and time. This constraint can be simplified to yield temperature as a function of the mass fractions of the species,

$$
T=\frac{h-\sum_{i=1}^{N} Y_{i} h_{i}^{f}}{c_{P}}+T^{o} .
$$

\section{II.C.2. Transformation to Reduced Variables}

Let us now transform the mass fractions into specific mole concentrations, $\hat{z}_{i}$, with

$$
\hat{z}_{i} \equiv \frac{Y_{i}}{\mathrm{M}_{i}}, \quad \text { for } i \in[1, N] .
$$

In specific mole variables it becomes more convenient to consider species thermodynamic properties in a molar basis, denoted by an overbar, while the mixture properties will remain in a mass basis. We can use the constraints in Eq. (18) to obtain a set of reduced variables. We choose the first $N-L$ species as our reduced variables,

$$
z_{i}=\hat{z}_{i}, \quad \text { for } i \in[1, N-L]
$$


We then couple the values of $\hat{z}_{i}$ for $i \in[1, N]$ to the values of $z_{i}$ by the linear relation

$$
\hat{z}_{i}(x, t)=\check{z}_{i}+\hat{\nu}_{i n} z_{n}(x, t), \quad \text { for } i \in[1, N] \text { and } n \in[1, N-L],
$$

where $\check{z}_{i}$ are the values of specific mole concentration when $z_{n}=0$ for all $n \in[1, N-L]$, and $\hat{\nu}_{\text {in }}$ is a coefficient matrix of dimension $N \times(N-L)$ that couples the variations in $z_{n}$ to $\hat{z}_{i}$. We define $\hat{\nu}_{i n}$ to be a full rank matrix that spans the column space of $\nu_{i j}$, where $\nu_{i j}$ need not be full rank. Therefore, $\sum_{i=1}^{N} \varphi_{l i} \hat{\nu}_{i n}=0$ for $l \in[1, L]$, and $n \in[1, N-L]$. This reduction is not unique, and the following is a technique to obtain $\hat{\nu}_{i n}$ : reduce the transpose of the $\nu_{i j}$ matrix to a row-echelon form, truncate the final $J-(N-L)$ rows of zeros in this echelon form, and take the transpose of this truncation to form $\hat{\nu}_{i n}$. In this construction of $\hat{\nu}_{i n}$, the first $N-L$ rows form a sub-matrix which is an $(N-L) \times(N-L)$ identity matrix, indicating that the reduced variables are the first $N-L$ species.

In this work we will consider two cases: i) the isothermal case, and ii) the adiabatic case. The enthalpy constraint in Eq. (7) reduces temperature to a rational function of polynomials of the reduced specific moles. Operating $\varphi_{l i}$ on Eq. (1), substituting Eq. (25) for mass fraction, and summing on $i$ species, yields the same $L$ constraints given in Eq. (18). The remaining $N-L$ ODEs are the governing equations in the reduced variables, which are

$$
\begin{aligned}
& \frac{\partial z_{i}}{\partial t}=\frac{\dot{\omega}_{i}\left(z_{n}, T\right)}{\rho}+\mathcal{D} \frac{\partial^{2} z_{i}}{\partial x^{2}}, \quad \text { for } i, n \in[1, N-L] \text {, } \\
& T= \begin{cases}\tilde{T}, & \text { if isothermal }, \\
\frac{h-\sum_{i=1}^{N} \hat{z}_{i}\left(z_{n}\right) \bar{h}_{i}^{f}}{\sum_{i=1}^{N} \hat{z}_{i}\left(z_{n}\right) \bar{c}_{P i}}+T^{o}, & \text { if adiabatic, }\end{cases}
\end{aligned}
$$

where there are no spatial variations in the species constraints as well as the temperature or enthalpy. Notice that $\dot{\omega}_{i}$ is now only a function of the reduced variables and temperature.

\section{II.C.3. Galerkin Reduction to ODEs}

To analyze Eq. (28), we apply a Galerkin projection onto an approximate inertial manifold (AIM). ${ }^{14}$ To accomplish this, we assume a spectral decomposition of

$$
z_{i}(x, t)=\sum_{m=0}^{\infty} z_{i, m}(t) \phi_{m}(x), \quad \text { for } i \in[1, N-L],
$$

where $z_{i, m}(t)$ is the $m^{t h}$ time-dependent amplitude associated with species $i$, and $\phi_{m}(x)$ are the corresponding basis functions. This projection is only in the reduced variables; our constraints, which must be constant in space, govern the evolution of the remaining species and temperature. We choose $\phi_{m}$ as the eigenfunctions of the diffusion operator, $\partial^{2} / \partial x^{2} \phi_{m}=-\mu_{m}^{2} \phi_{m}$, that match the boundary conditions, which gives us the complete basis

$$
\phi_{m}=\cos \left(\frac{m \pi x}{\ell}\right), \quad \text { for } m \in[0, \infty) .
$$

These basis functions are orthogonal, $\left\langle\phi_{m}, \phi_{n}\right\rangle=0, n \neq m$, and their eigenvalues are real, given by $\mu_{m}=m \pi / \ell$. By substituting Eq. (30) into Eq. (28), taking the inner product with each basis function, $\phi_{n}$, and taking advantage of the orthogonality of the basis functions, we obtain an infinite system of ODEs for the evolution of the amplitudes

$$
\frac{d z_{i, m}}{d t}=\frac{<\phi_{m}, \dot{\omega}_{i}\left(\sum_{n=0}^{\infty} z_{i, n} \phi_{n}\right) / \rho>}{<\phi_{m}, \phi_{m}>}-\mathcal{D} \mu_{m}^{2} z_{i, m}, \quad \text { for } i \in[1, N-L], \text { and } m \in[0, \infty),
$$

where the initial conditions of the amplitudes are given by

$$
\tilde{z}_{i, m}=\frac{<\phi_{m}, \tilde{Y}_{i} / \mathrm{M}_{i}>}{<\phi_{m}, \phi_{m}>}, \quad \text { for } i \in[1, N-L], \text { and } m \in[0, \infty)
$$


For our choice of basis functions, $<\phi_{m}, \phi_{m}>=\ell$ for $m=0$ and $<\phi_{m}, \phi_{m}>=\ell / 2$ for $m \geq 1$. This leaves the system of ODEs as

$$
\begin{gathered}
\frac{d z_{i, 0}}{d t}=\underbrace{\frac{1}{\ell} \int_{0}^{\ell} \frac{\dot{\omega}_{i}\left(z_{n}(x, t)\right)}{\rho} d x}_{\equiv \dot{\omega}_{i, 0}}, \quad \text { for } i, n \in[1, N-L], \\
z_{i, 0}(0)=\tilde{z}_{i, 0}=\underbrace{\frac{1}{\ell \mathrm{M}_{i}} \int_{0}^{\ell} \tilde{Y}_{i}(x) d x, \quad \text { for } i \in[1, N-L],}_{\equiv \dot{\omega}_{i, m}} \\
\frac{d z_{i, m}}{d t}=\underbrace{\frac{2}{\ell} \int_{0}^{\ell} \frac{\dot{\omega}\left(z_{n}(x, t)\right)}{\rho} \cos \left(\frac{m \pi x}{\ell}\right) d x}_{z_{i, m}(0)=\tilde{z}_{i, m}=}-\frac{\pi^{2} m^{2} \mathcal{D}}{\ell^{2}} z_{i, m}, \\
\text { for } i, n \in[1, N-L], \text { and } m \in[1, \infty),
\end{gathered}
$$

For small $\ell$ and $m \geq 1$, the diffusion term dominates the reaction term, which makes the $m \geq 1$ amplitudes decay rapidly. The integration of the $\dot{\omega}_{i, m}$ terms from Eqs. (34) and (36) can be performed analytically in the isothermal case for our simple choice of basis functions; however, for the adiabatic case or more complex basis functions this integration must be calculated at every time step using numerical quadrature.

We define a diffusion time scale,

$$
\tau_{\mathcal{D}} \equiv \frac{\ell^{2}}{\pi^{2} \mathcal{D}}
$$

Truncating at a finite $M$ reduces the infinite system of ODEs in Eqs. (34) and (36) to the system of $(M+1)(N-L)$ ODEs,

$$
\begin{aligned}
\frac{d z_{i, m}}{d t}=\dot{\omega}_{i, m}-\frac{m^{2}}{\tau_{\mathcal{D}}} z_{i, m}, & \text { for } i \in[1, N-L], \text { and } m \in[0, M], \\
z_{i, m}(0)=\tilde{z}_{i, m}, & \text { for } i \in[1, N-L], \text { and } m \in[0, M] .
\end{aligned}
$$

For very small $\ell$, a truncation at $M=0$ is appropriate in which case Eq. (28) reduces to a spatially homogeneous system,

$$
\begin{aligned}
\frac{d z_{i}}{d t} & =\dot{\omega}_{i}\left(z_{n}, T\right), & & \text { for } i, n \in[1, N-L], \\
z_{i}(0) & =\tilde{z}_{i}, & & \text { for } i, n \in[1, N-L] .
\end{aligned}
$$

For this case $\tau_{\mathcal{D}}$ is very small; therefore, the diffusion term will dominate the reaction term. This will make the diffusion infinitely fast, all spatial inhomogeneities equilibrate in an infinitesimal time, and a spatially homogeneous system will be recovered.

\section{Zel'dovich Reaction Mechanism}

We evaluate the Zel'dovich mechanism of $N O$ production similar to the system studied by Al-Khateeb et al. ${ }^{12}$ (see Table 1 ). This system has $N=5$ species and $J=2$ reactions. Conservation of atomic elements

\begin{tabular}{|c|c|c|c|c|}
\hline$j$ & Reaction & $a_{j}$ & $\beta_{j}$ & $\bar{E}_{j}$ \\
\hline 1 & $N+N O \leftrightharpoons N_{2}+O$ & $2.107 \times 10^{13}$ & 0.00 & 0.0 \\
2 & $N+O_{2} \leftrightharpoons N O+O$ & $5.839 \times 10^{9}$ & 1.01 & 6196 \\
\hline
\end{tabular}

Table 1. The Zel'dovich mechanism for $N O$ production. The units for a are $\mathrm{mol} /(\mathrm{cms} K), \bar{E}$ are $\mathrm{cal} / \mathrm{mol}$, and $\beta$ is dimensionless. 
yields two algebraic constraints, while a third algebraic constraint for conservation of molecules is obtained since the system is comprised of exclusively bimolecular reactions. This leaves $L=3$ species constraints that allow the system to be reduced to $N-L=2$ reduced variables; we choose to evaluate the specific moles of $N O$ and $N$ as $z_{1}$ and $z_{2}$, respectively. The evolution of the three remaining species $N_{2}, O$, and $O_{2}$, identified as $\hat{z}_{3}, \hat{z}_{4}$ and $\hat{z}_{5}$, respectively, are coupled to the evolution of $z_{1}$ and $z_{2}$ by algebraic constraints. This leaves us with the reaction-species coefficient, species constraint, and species reduction matrices as

$$
\nu_{i j}=\left[\begin{array}{rr}
-1 & 1 \\
-1 & -1 \\
1 & 0 \\
1 & 1 \\
0 & -1
\end{array}\right], \quad \varphi_{l i}=\left[\begin{array}{lllll}
1 & 1 & 1 & 1 & 1 \\
1 & 1 & 2 & 0 & 0 \\
1 & 0 & 0 & 1 & 2
\end{array}\right], \quad \hat{\nu}_{i j}=\left[\begin{array}{rr}
1 & 0 \\
0 & 1 \\
-\frac{1}{2} & -\frac{1}{2} \\
0 & -1 \\
-\frac{1}{2} & \frac{1}{2}
\end{array}\right]
$$

respectively. For this system $\nu_{i j}$, dimension $N \times J$, happens to be full rank and therefore the same dimension as $\hat{\nu}_{i j}$, dimension $N \times(N-L)$; this is not generally the case. For all cases, the system is taken to be isobaric at $P_{o}=1.6629$ bar. The system constraints given by Eq. (18) are set uniform in space with values that correspond to when all five species consist of $10^{-3} \mathrm{~mol}$. The constraints form the following algebraic relationships for the full species concentrations as functions of reduced species

$$
\left[\begin{array}{c}
\hat{z}_{1}(x, t) \\
\hat{z}_{2}(x, t) \\
\hat{z}_{3}(x, t) \\
\hat{z}_{4}(x, t) \\
\hat{z}_{5}(x, t)
\end{array}\right]=\left[\begin{array}{c}
0 \\
0 \\
1.6663 \times 10^{-2} \\
1.6663 \times 10^{-2} \\
8.3317 \times 10^{-3}
\end{array}\right] \mathrm{mol} / g+\left[\begin{array}{rr}
1 & 0 \\
0 & 1 \\
-\frac{1}{2} & -\frac{1}{2} \\
0 & -1 \\
-\frac{1}{2} & \frac{1}{2}
\end{array}\right] \cdot\left[\begin{array}{c}
z_{1}(x, t) \\
z_{2}(x, t)
\end{array}\right]
$$

For the isothermal case the temperature is taken to be $T=4000 \mathrm{~K}$; this case also yields an isochoric reaction with a fixed volume of $V=10^{3} \mathrm{~cm}^{3}$. The species' Gibbs free energy is obtained directly from the polynomial fit in the CHEMKIN thermodynamics database. ${ }^{15}$

For the adiabatic case we shift to a model for Gibbs free energy with a constant specific heat assumption,

$$
\bar{g}_{i}^{o}=\underbrace{\bar{h}_{i}^{f}+\bar{c}_{P i}\left(T-T^{o}\right)}_{\bar{h}_{i}}-\underbrace{\left(\bar{s}_{i}^{f}+\bar{c}_{P i} \log \left(\frac{T}{T^{o}}\right)\right)}_{\bar{s}_{i}^{o}} T
$$

where for $i=1, \ldots, N, \bar{h}_{i}, \bar{s}_{i}^{f}$, and $\bar{s}_{i}^{o}$ are the enthalpy, entropy at reference temperature and pressure, and the entropy at reference pressure for species $i$, respectively. This change was made in order to maintain linear thermodynamic properties outside of the temperature range of the CHEMKIN polynomial fit. The values of $\bar{c}_{P i}, \bar{h}_{i}^{f}$ and $\bar{s}_{i}^{f}$ are calculated using a least squares fit of the CHEMKIN polynomial fits and are listed in Table 2. For the adiabatic case the enthalpy is chosen to be $h=9.0376 \times 10^{10} \mathrm{erg} / \mathrm{g}$, such that the physical equilibrium remains at $T=4000 \mathrm{~K}$ and $V=10^{3} \mathrm{~cm}^{3}$; however, this system is not isochoric, and the volume changes as the system evolves.

\begin{tabular}{|c|c|c|r|c|}
\hline & Isothermal & \multicolumn{3}{|c|}{ Adiabatic } \\
\hline$i$ & $\bar{g}_{i}^{o}$ & $\bar{c}_{P i}$ & $\bar{h}_{i}^{f}$ & $\bar{s}_{i}^{f}$ \\
\hline 1 & $-9.7276 \times 10^{12}$ & $3.6774 \times 10^{8}$ & $8.6712 \times 10^{11}$ & $2.0399 \times 10^{9}$ \\
2 & $-2.7915 \times 10^{12}$ & $2.1120 \times 10^{8}$ & $4.7223 \times 10^{12}$ & $1.5274 \times 10^{9}$ \\
3 & $-9.8012 \times 10^{12}$ & $3.6167 \times 10^{8}$ & $-4.0402 \times 10^{10}$ & $1.8421 \times 10^{9}$ \\
4 & $-5.3580 \times 10^{12}$ & $2.1002 \times 10^{8}$ & $2.4922 \times 10^{12}$ & $1.6130 \times 10^{9}$ \\
5 & $-1.0455 \times 10^{12}$ & $3.8825 \times 10^{8}$ & $-5.1316 \times 10^{10}$ & $1.9599 \times 10^{9}$ \\
\hline
\end{tabular}

Table 2. Thermodynamic data for the Zel'dovich mechanism. The units for $\bar{c}_{P}$ and $\bar{s}^{f}$ are $\mathrm{erg} /(\mathrm{mol} \mathrm{K})$, and $\bar{g}^{o}$ and $\bar{h}^{f}$ are $\mathrm{erg} / \mathrm{mol}$. 


\section{Results and Discussion}

\section{IV.A. Spatially Homogeneous}

\section{IV.A.1. Isothermal System}

We start by examining the isothermal system for the spatially homogeneous, $M=0$, case, which is governed by the equations

$$
\begin{aligned}
& \frac{d z_{1}}{d t}=\alpha_{1}+\alpha_{2} z_{1}+\alpha_{3} z_{2}+\alpha_{4} z_{1} z_{2}+\alpha_{5} z_{2}^{2} \\
& \frac{d z_{2}}{d t}=\alpha_{6}+\alpha_{7} z_{1}+\alpha_{8} z_{2}+\alpha_{9} z_{1} z_{2}+\alpha_{10} z_{2}^{2}
\end{aligned}
$$

where the system's coefficients are given in Table 3. To identify the branches of the SIM, we first must

\begin{tabular}{|c|c|c|c|c|}
\hline$\alpha_{1}$ & $\alpha_{2}$ & $\alpha_{3}$ & $\alpha_{4}$ & $\alpha_{5}$ \\
\hline $250.5 \mathrm{~mol} /(\mathrm{g} \mathrm{s})$ & $-997301 / \mathrm{s}$ & $1.1611 \times 10^{7} 1 / \mathrm{s}$ & $-3.2210 \times 10^{9} \mathrm{~g} /(\mathrm{mol} \mathrm{s})$ & $6.986 \times 10^{8} \mathrm{~g} /(\mathrm{mol} \mathrm{s})$ \\
\hline \hline$\alpha_{6}$ & $\alpha_{7}$ & $\alpha_{8}$ & $\alpha_{9}$ & $\alpha_{10}$ \\
\hline $250.5 \mathrm{~mol} /(\mathrm{g} \mathrm{s})$ & $847001 / \mathrm{s}$ & $-1.1656 \times 10^{7} 1 / \mathrm{s}$ & $-1.8359 \times 10^{9} \mathrm{~g} /(\mathrm{mol} \mathrm{s})$ & $-6.977 \times 10^{8} \mathrm{~g} /(\mathrm{mol} \mathrm{s})$ \\
\hline
\end{tabular}

Table 3. Coefficients of the isothermal spatially homogeneous Zel'dovich mechanism.

find the system's equilibria via an algebraic method. Using a local linear analysis, we characterize these equilibria as sources, saddles, and sinks. Then, we search for heteroclinic orbits that connect the unstable eigenvector of a saddle equilibrium that has only one positive eigenvalue to the physical equilibrium sink along the eigenvector associated with its slowest eigenvalue. The saddle equilibria can also be located at infinity; they can be found using projective space or Poincaré sphere mappings. ${ }^{11,12}$

We see from Eqs. (46) and (47) that the isothermal systems' reaction source terms, $\dot{\omega}_{i}$, are polynomials. This allows one to use a homotopy continuation technique to identify all of the equilibria of the polynomial system. ${ }^{16,17}$ We find that our system has three finite equilibria, shown as black dots in Fig. 1, including the

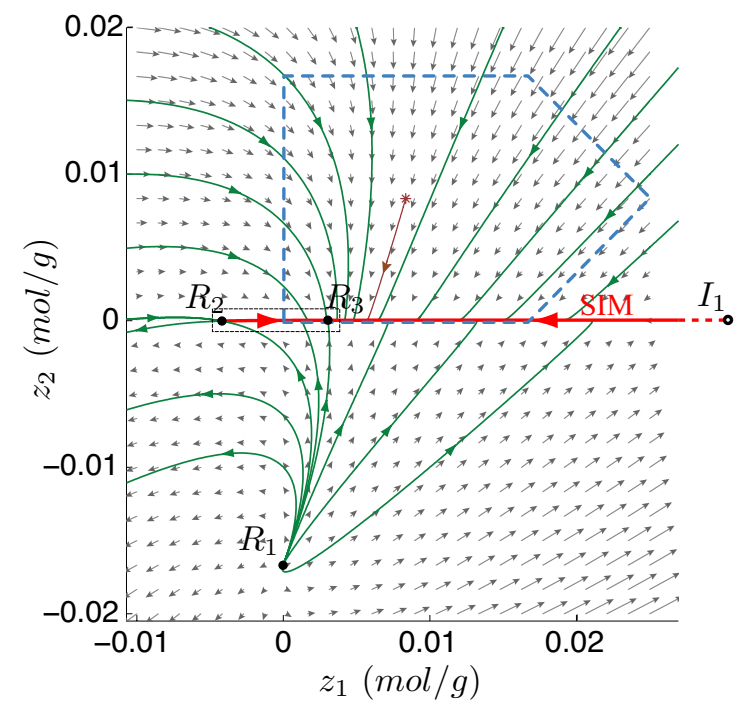

(a)

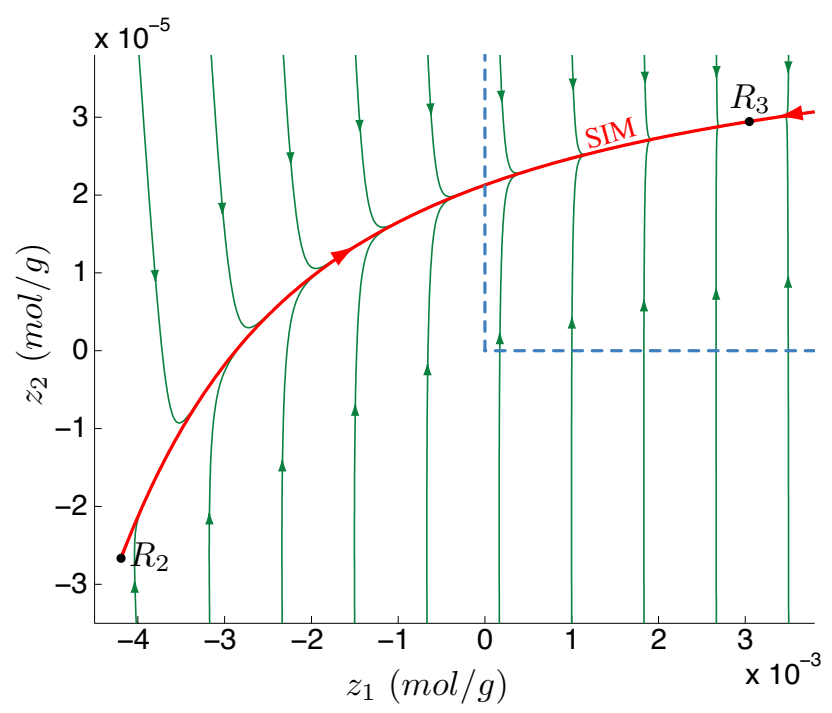

(b)

Figure 1. The spatially homogeneous SIM for the isothermal Zel'dovich mechanism.

physical equilibrium sink, labeled $R_{3}$, at

$$
z_{i, R_{3}}=\left\{3.0474 \times 10^{-3}, 2.9446 \times 10^{-5}\right\} \mathrm{mol} / \mathrm{g} .
$$

The Jacobian of the linearized system about this equilibrium has eigenvalues

$$
\lambda_{i, R_{3}}=\left\{-1.7295 \times 10^{7},-1.9129 \times 10^{5}\right\} 1 / s .
$$


We associate the reciprocal of each eigenvalue with a timescale of the system, $\tau_{i}=\left|1 / \lambda_{i}\right|$, and therefore label the first eigenvalue "fast" with $\tau_{1}=5.78 \times 10^{-8} \mathrm{~s}$ and the second one "slow" with $\tau_{2}=5.23 \times 10^{-6} \mathrm{~s}$.

To calculate the SIM, we search for a non-physical equilibrium with a saddle character. This system's other equilibria include the source, $R_{1}$, and the saddle, $R_{2}$. The saddle has a heteroclinic orbit emanating parallel to its unstable eigenvector that connects to the slow eigenvector of the physical equilibrium; this trajectory is one branch of the SIM, and is shown in thick red in Fig. 1. The other branch of the SIM originates at an equilibrium at infinity, labeled $I_{1}$. Infinite equilibria are beyond the scope of this paper; more details are available in Davis and Skodje ${ }^{11}$ and Al-Khateeb et al. ${ }^{12}$ Fig. 1a shows the entirety of the physical domain, bounded by a blue dashed line. The gray arrows indicate the magnitude and direction of change of the state variables in phase space, and the green lines show various trajectories. The thin black dashed line shows the boundaries of the detailed view in Fig. 1b which shows the branch of the SIM connecting $R_{2}$ to $R_{3}$. The selected trajectories show why the SIM is important as a reduction technique; as the trajectories evolve, they converge to become exponentially close to the SIM in a finite amount of time. The brown line in Fig. 1a shows the trajectory associated with the time evolution of species for the case shown in the time domain in Fig. 2. This case has the initial conditions of $z_{1}=z_{2}=8.3317 \times 10^{-3} \mathrm{~mol} / \mathrm{g}$. Two time scales are evident in Fig. 2; the fast, which occurs at approximately $10^{-7} \mathrm{~s}$, is the evolution

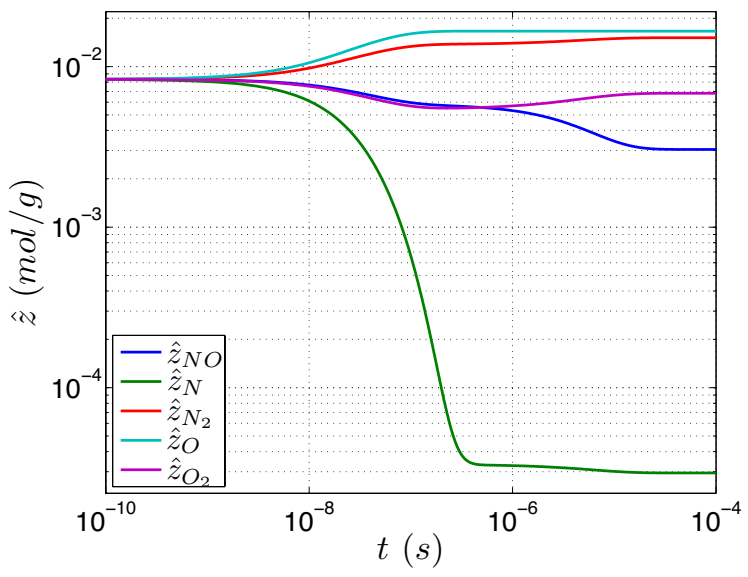

Figure 2. Spatially homogeneous isothermal system evolution of species.

toward the SIM, while the slow, which occurs at approximately $10^{-5} s$, is the evolution along the SIM toward equilibrium. Notice that these time scales correspond closely to the time scales at equilibrium.

\section{IV.A.2. Adiabatic System}

To evaluate an adiabatic system, the technique is the same, except $\dot{\omega}_{i}$ now has a variable temperature. The reaction source term can be shown to be a polynomial of infinite degree, which admits an infinite number of equilibria and renders the homotopy continuation techniques unable to find the roots of the system. This presents a problem, since in order to construct the SIM, we must find the saddle equilibria that connect by heteroclinic orbit to the physical equilibrium sink. The physical equilibrium can be located by a minimization of thermodynamic potentials, among other methods; the system's other equilibria are more difficult to find. The method we employ is to vary the temperature incrementally and solve for the equilibrium of the isothermal system at each temperature. We then check the enthalpy at each isothermal equilibrium to see if it matches the adiabatic constraint. This technique does not yield all equilibria of the system; however, for the systems we have examined, the saddle equilibria from which the SIM originates migrates only a small distance in phase space.

For our system the physical equilibrium is located at

$$
z_{i, R_{3}^{\prime}}=\left\{3.0054 \times 10^{-3}, 2.9862 \times 10^{-5}\right\} \mathrm{mol} / \mathrm{g} .
$$

There is a shift of approximately $1.4 \%$ in the location of the physical equilibrium from the isothermal case. This is due to the change in the modeling of the Gibbs free energy. 
The dynamics in the near neighborhood of the equilibrium also change slightly. This can be seen by evaluating the eigenvalues of the Jacobian matrix, which are now

$$
\lambda_{i, R_{3}^{\prime}}=\left\{-1.7628 \times 10^{7},-2.2181 \times 10^{5}\right\} 1 / s .
$$

Again, we label the first eigenvalue "fast" with $\tau_{1}=5.67 \times 10^{-8} \mathrm{~s}$ and the second one "slow" with $\tau_{2}=$ $4.51 \times 10^{-6} \mathrm{~s}$.

To find the SIM of the adiabatic system, we must locate the saddle equilibria from which the SIM originates. We do this by tracking the three finite equilibria for an isothermal system with varying temperatures and track the enthalpy of those equilibria. When the enthalpy of the isothermal equilibria matches the enthalpy constraint on the adiabatic system, the adiabatic system will have an equilibrium at that location and temperature. This is shown graphically in Fig. 3. Fig. 3a shows the locus of the isothermal equilibria in

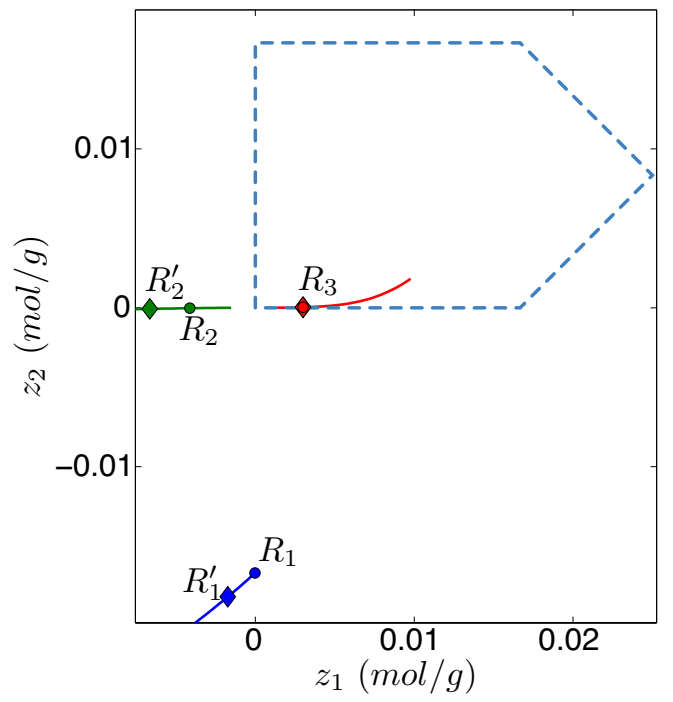

(a)

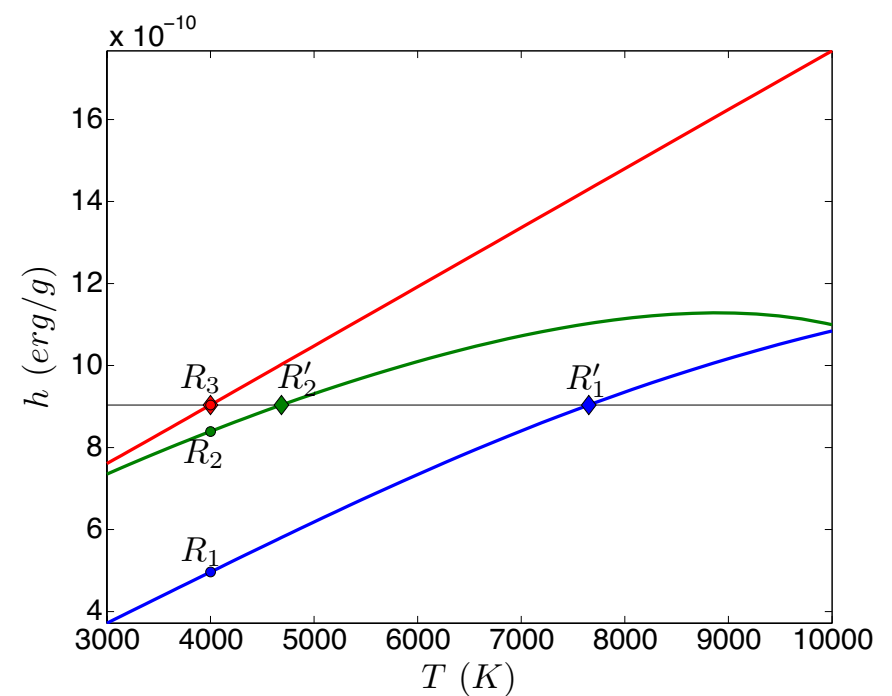

(b)

Figure 3. Isothermal equilibria for varying temperatures.

phase space, while Fig. 3b shows the enthalpy of each equilibrium as a function of specified system temperature. We see from Fig. 3a that the equilibria $R_{1}^{\prime}$ and $R_{2}^{\prime}$ shift from their locations in the isothermal system to new locations in the adiabatic system. Notice in Fig. 3b that the enthalpy of the isothermal equilibria does not monotonically increase with temperature. This highlights the fact that additional equilibria are admitted, because the path of each isothermal equilibria could cross the specified enthalpy many more times as the temperature is varied, yielding additional adiabatic equilibria.

When we evaluate the eigenvalues of the Jacobian of the shifted equilibria, we find that $R_{1}^{\prime}$ and $R_{2}^{\prime}$ retain their characters as source and saddle, respectively. Also, the heteroclinic orbit from $R_{2}^{\prime}$ along the unstable eigenvector still connects to $R_{3}$ along its slow eigenvector, making it one branch of the SIM. The other branch of the SIM now no longer originates at infinity, but has shifted to originate at the pole $P_{1}^{\prime}$, which can be shown to be a first order pole. This pole is located where the line $z_{2}=0$ crosses the line $T=0$.

The phase space of the adiabatic system is shown in Fig. 4. In Fig. 4a we see the phase space that includes the entire physical domain. Here we see the shifted equilibria and both SIM branches, which are the heteroclinic orbits that connect $R_{2}^{\prime}$ and $P_{1}^{\prime}$ to $R_{3}$. Notice the line identified as $T=0$ in the upper right hand corner of the phase space. This is the location in phase space where the enthalpy constraint yields a zero temperature. To the upper right past this line, the temperatures are negative, and the time rate of change of the species mole fraction becomes imaginary; because of this we restrict our evaluation to regions of phase space where $T \geq 0$. In Fig. $4 \mathrm{~b}$ we see an expanded view of the branch of the SIM that connects $R_{2}^{\prime}$ to $R_{3}$.

An example of the time evolution of species for the adiabatic case, which corresponds to the brown trajectory in Fig. 4a, is shown in Fig. 5. Again, the initial conditions are $z_{1}=z_{2}=8.3317 \times 10^{-3} \mathrm{~mol} / \mathrm{g}$, which now corresponds to a temperature of $T=2156.8 \mathrm{~K}$. The species evolution is shown in Fig. $5 \mathrm{a}$, while the temperature is shown in Fig. 5b. Again, two time scales are evident in Fig. 5; the evolution 


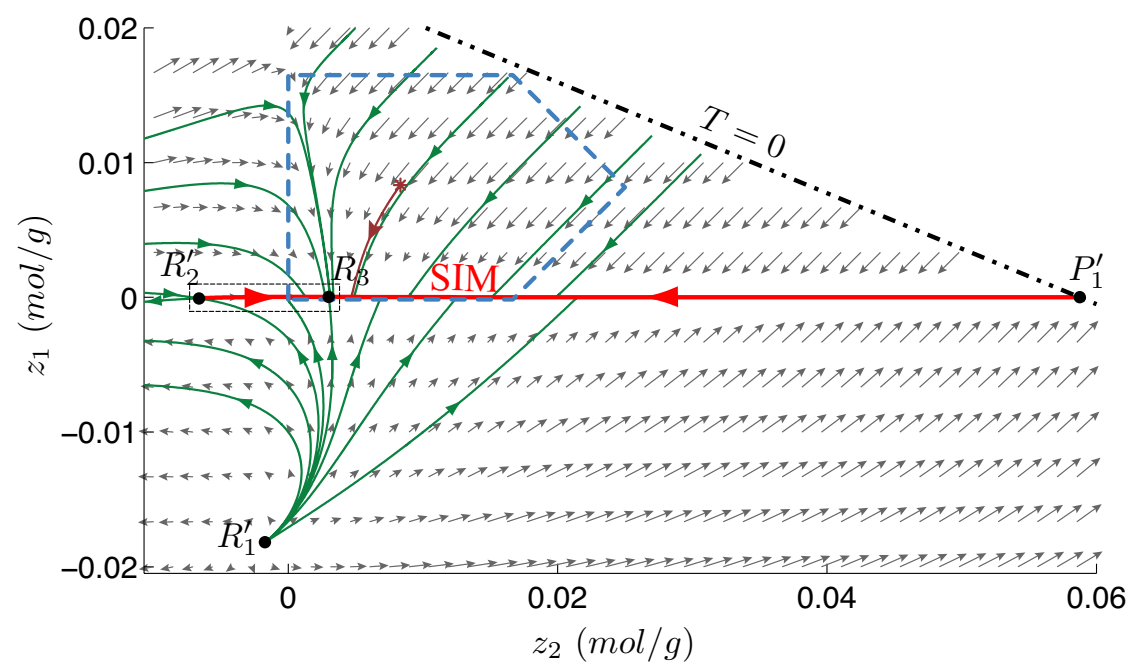

(a)

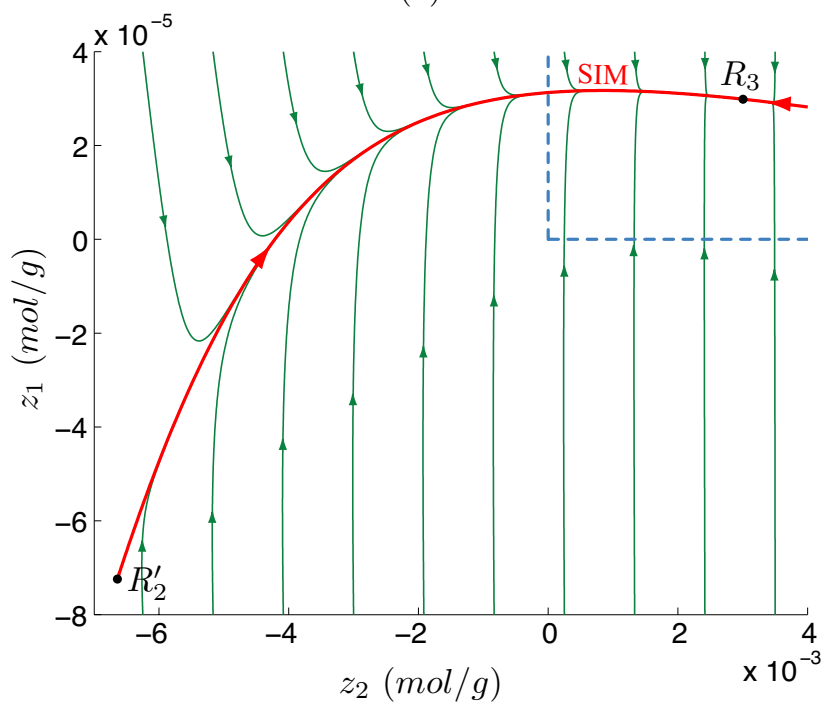

(b)

Figure 4. The spatially homogeneous SIM for the adiabatic Zel'dovich mechanism.

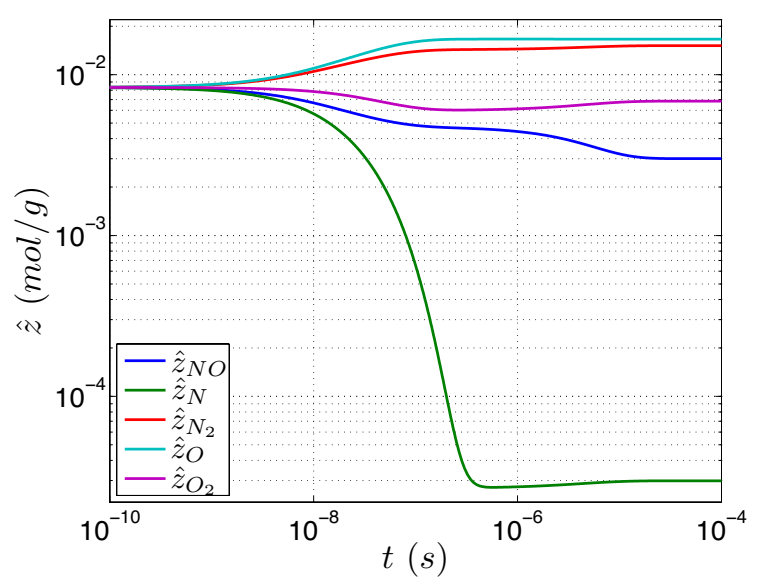

(a)

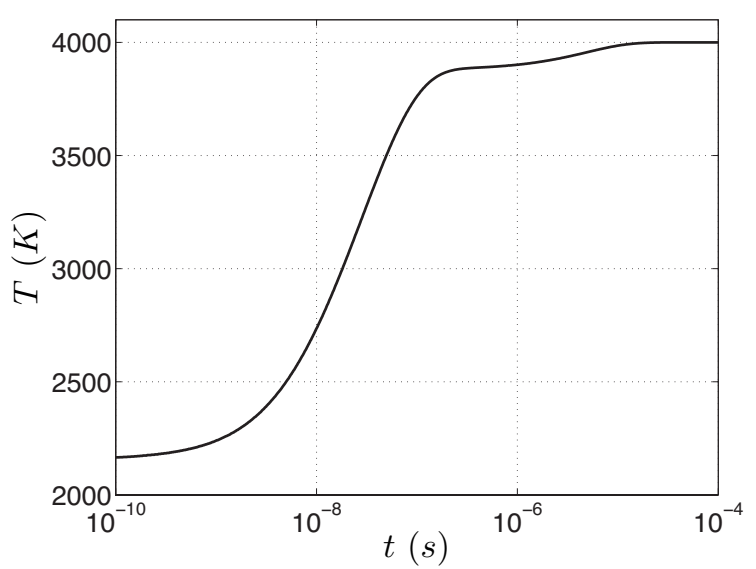

(b)

Figure 5. Evolution of the spatially homogeneous adiabatic system. 
toward the SIM occurs around $10^{-7} s$, which correlates with the fast time scale, and the evolution along the SIM toward equilibrium, which occurs around $10^{-5} s$, correlates with the slow time scale. The adiabatic results are compared with the isothermal case, shown in Fig. 2, where each case shows the same initial species concentrations and equilibria. While there are no major differences in this evolution, we do see that changing the constraint from isothermal to adiabatic has changed the dynamics, and therefore changed the paths through phase space.

\section{IV.B. Diffusion Correction}

We next examine an isothermal spatially inhomogeneous system to evaluate changes in the SIM for systems with small correction for fast finite diffusion. To maintain full resolution of the spatio-temporal dynamics for this choice of $M$, we restrict our analysis to a sufficiently fast diffusion time scale, or short length scale.

Here we note that the time evolution of the $z_{i, 0}$ term remains identical to the spatially homogeneous system when $z_{i, m}=0$ for all $i$ and all $m \geq 1$. This means that the entire spatially homogeneous phase space, including the spatially homogeneous equilibria, remains as a subspace in the diffusion-modified system's phase space. The eigenvalues of the Jacobian linearized about these spatially homogeneous equilibria are modified in the diffusion-corrected space such that they can be described analytically. The phase space has increased in dimension from $N-L$ to $(M+1)(N-L)$, meaning that there are $M+1$ times as many eigenvalues as in the spatially homogeneous case. The modified eigenvalues are given by

$$
\lambda_{i, m}=\lambda_{i}-\frac{m^{2}}{\tau_{\mathcal{D}}}, \quad \text { for } i \in[1, N-L], \text { and } m \in[0, M],
$$

where $\lambda_{i}$ are the eigenvalues of the spatially homogeneous equilibria. This means that the additional modes of the spatially homogeneous equilibria for the diffusion-correction are always more negative, or more stable.

For the present analysis, we truncate this series at $M=1$ and choose a diffusion time scale, $\tau_{\mathcal{D}}=$ $2 \times 10^{-8} s$, to be somewhat faster than the fast spatially homogeneous reaction time scale in the neighborhood of $R_{3}$. This justifies our truncation at $M=1$ and will display the effects of a small amount of diffusion in our system. Since $\mathcal{D}=14 \mathrm{~cm}^{2} / \mathrm{s}$ for this mechanism, our choice of $\tau_{\mathcal{D}}$ gives a length scale of $\ell=17 \mu \mathrm{m}$. The value of $\mathcal{D}$ is approximated from an average of the ordinary multicomponent diffusion coefficients in the CHEMKIN TRANSPORT database. ${ }^{18}$ In Fig. 6 we show the reaction (solid lines) and first correction for diffusion (dashed lines) time scales in the neighborhood of $R_{3}$, where the time scales are defined as $\tau_{i, m}=\left|1 / \lambda_{i, m}\right|$. From this

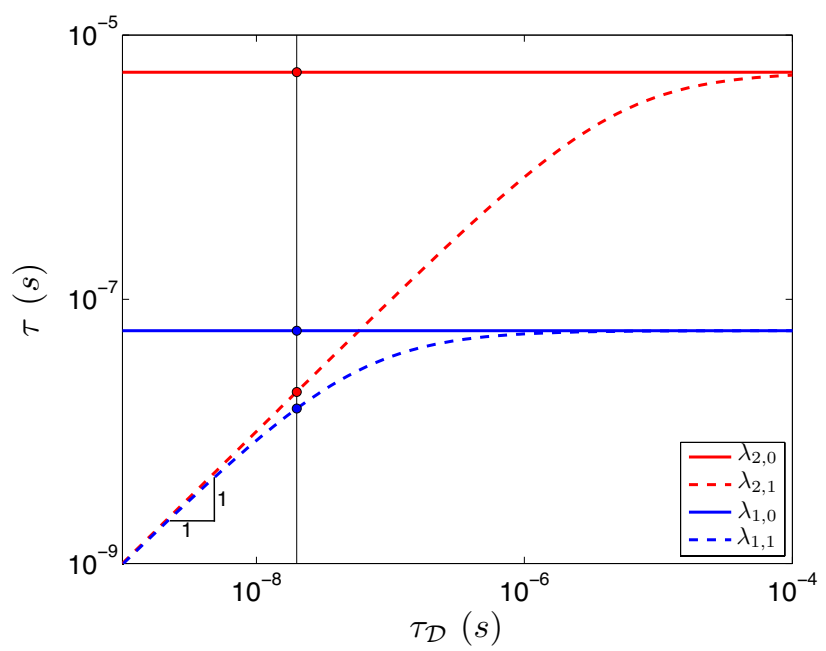

Figure 6. The time scales of the Galerkin projection of the Zel'dovich mechanism as a function of diffusion time scale.

diagram we can evaluate the limits of $\tau_{\mathcal{D}}$. In the limit of $\tau_{\mathcal{D}} \rightarrow 0$, which idealizes systems where $\ell \rightarrow 0$, diffusion is infinitely fast. This makes all variations in space diffuse instantaneously, and the system behaves as spatially homogeneous globally. For systems in the limit of $\ell \rightarrow \infty$ our current analysis is insufficient. For these systems our current definition of $\tau_{\mathcal{D}} \rightarrow \infty$ indicates infinitely slow diffusion, which is only accurate for the fundamental mode. A truncation at $M=1$ for systems with long length would neglect the modes where diffusion is important. To fully resolve systems with long length, additional modes must be considered. 
In Fig. 7 we see a three dimensional projection of the four dimensional phase space for this problem. The vertical axis shows the $z_{1,1}$ concentration, while the horizontal axes show the two spatially homogeneous

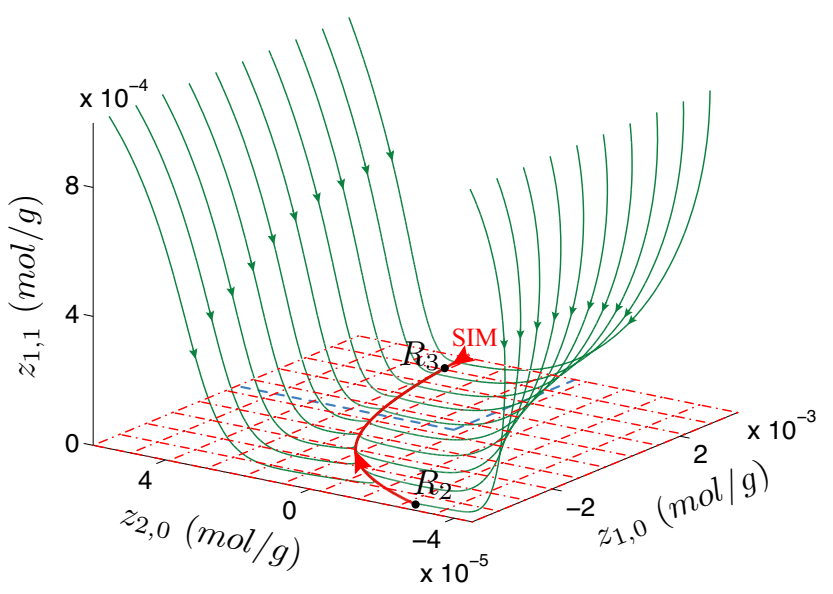

Figure 7. The diffusion correction SIM for the isothermal Zel'dovich mechanism.

concentrations; $z_{2,1}$ is not depicted in this figure, but decays in a similar fashion to $z_{1,1}$. The $z_{1,0}$ and $z_{2,0}$ axis are scaled similar to Fig. 1b; in fact the two dimensional projection of the $z_{1,0^{-}} z_{2,0}$ plane is almost identical to that figure. Fig. 7 shows $R_{3}$, which is the physical equilibrium sink, and $R_{2}$, which is a saddle equilibrium with one positive eigenvalue. Both of these equilibria are spatially homogeneous $\left(z_{i, m}=0\right.$ for all $i$ and $m \geq 1$ ), and there is a heteroclinic orbit that connects the unstable eigenvector of $R_{2}$ to the slow eigenvector of $R_{3}$, making it a branch of the one-dimensional SIM. The other branch of the SIM still originates at the spatially homogeneous equilibrium $I_{1}$ at infinity. The SIM we have calculated for this diffusion correction system is identical to the SIM for the spatially homogeneous isothermal system. For our choice of a fast $\tau_{\mathcal{D}}$, the slow dynamics, and therefore the SIM, exist entirely in the spatially homogeneous subspace of the system. This is seen since the equilibria from which the SIM branches originate, as well as the eigenvectors along which the SIM branches initially propagate, all exist in the spatially homogeneous subspace. Notice in Fig. 7 that the diffusion correction amplitudes decay faster than the spatially homogeneous (reaction only) amplitudes. In fact, the spatially homogeneous subspace, the $z_{1,0}-z_{2,0}$ plane, is a two-dimensional SIM for this problem, meaning only the fastest dynamics involve diffusion processes. We can again see this by examining the evolution of the amplitudes, which is shown in Fig. 8a. In Fig. 8a we see the solid lines

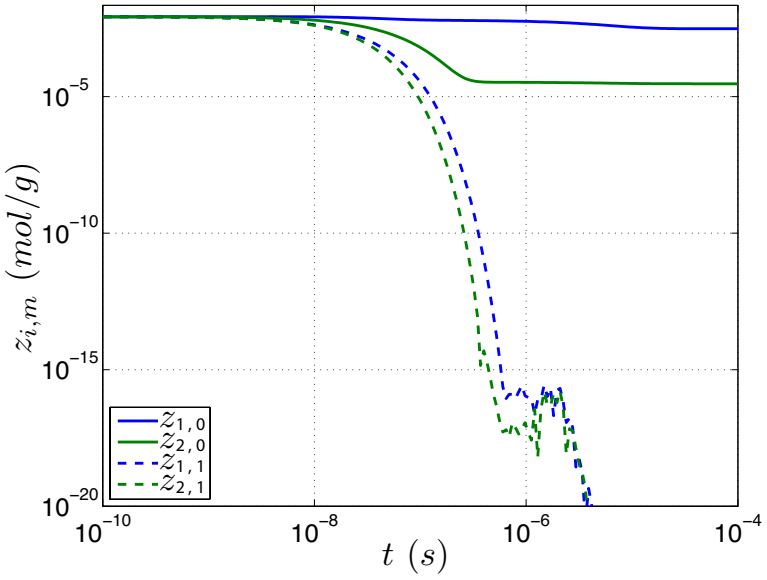

(a)

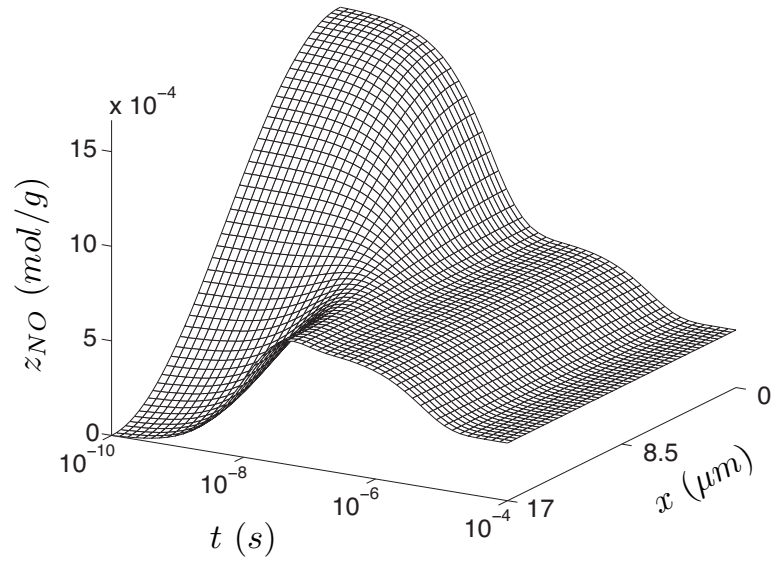

(b)

Figure 8. Evolution of isothermal diffusion correction system.

depicting the spatially homogeneous amplitudes and the dashed lines the diffusion correction amplitudes. The initial conditions are $z_{i, m}=8.3317 \times 10^{-3}(\mathrm{~mol} / \mathrm{g})$ for all $i$ and $m$; these initial conditions are atypical. An actual initial value problem is more likely to have initial amplitudes whose values are set by Fourier decomposition of the initial conditions and will likely contain information at all frequencies. For the initial 
conditions in Fig. 8a, the spatially homogeneous amplitudes evolve almost identically to their corresponding values in Fig. 2. The diffusion correction amplitudes begin to decay spectrally to machine precision zero before the reaction begins to affect the amplitudes. This is because of our choice of a fast $\tau_{\mathcal{D}}$. Considering slower values of $\tau_{\mathcal{D}}$, there is a threshold where the spatially homogeneous subspace of the system no longer is a two-dimensional SIM. Beyond this threshold the fast dynamics will involve a stronger coupling of reaction and diffusion dynamics. For slower yet values of $\tau_{\mathcal{D}}$, the SIM itself will bifurcate out of the spatially homogeneous subspace, and all dynamics, including the slow dynamics, will involve a coupling of reaction and diffusion processes. In Fig. 8b we see the reconstructed spatio-temporal evolution of the species $z_{N O}$ for the initial conditions in Fig. 8a. The spatial variations decay at a faster time scale than the spatially homogeneous or reaction kinetics occur.

\section{Conclusions}

We have implemented two important extensions by developing SIMs for: i) adiabatic systems, and ii) spatially inhomogeneous systems with a simple diffusion model. We have successfully identified a SIM for an adiabatic spatially homogeneous system. In doing so, we have displayed a technique to track non-isothermal equilibria by varying the temperature of isothermal system to satisfy system constraints. The adiabatic system examined displayed similarities to its analogous isothermal system, but showed different dynamics that are important to capture in a reduction technique.

For the diffusion correction example, when the length scales are small enough to make the diffusion time scale faster than the fast reaction time scale, the effects of diffusion on a reactive system are minimal, allowing for a global analysis of the system. When lengths are near or above a critical length where the diffusion time scale is on the same order as the slow reaction time scale, diffusion will play a more important role. Because of our choice of a small length scale and a fast diffusion time scale, we could examine a global scale diffusion correction. This allowed us to maintain spatial resolution with a truncation that included a single correction for diffusion. In the limit of large length scales, the present analysis that only models the fundamental mode is inadequate; the system would require an inclusion of additional modes to fully resolve the dynamics.

\section{Acknowledgments}

The authors recognize the support of the National Science Foundation (NSF) under Grant No. CBET0650843. J. D. Mengers also recognizes the support from the University of Notre Dame Department of Applied and Computational Mathematics and Statistics fellowship.

\section{References}

\footnotetext{
${ }^{1}$ Griffith, J. F., "Reduced kinetic-models and their application to practical combustion systems," Progress in Energy and Combustion Science, Vol. 21, No. 1, 1995, pp. 25-107.

${ }^{2} \mathrm{Lu}$, T. F. and Law, C. K., "Toward accommodating realistic fuel chemistry in large-scale computations," Progress in Energy and Combustion Science, Vol. 35, No. 2, 2009, pp. 192-215.

${ }^{3}$ Singh, S., Powers, J. M., and Paolucci, S., "On slow manifolds of chemically reactive systems," Journal of Chemical Physics, Vol. 117, No. 4, 2002, pp. 1482-1496.

${ }^{4}$ Ren Z. and Pope, S. B., "Transport-chemistry coupling in the reduced description of reactive flows," Combustion Theory and Modeling, Vol. 11, No. 5, 2007, pp. 715-739.

${ }^{5}$ Davis, M. J., "Low-dimensional manifolds in reaction-diffusion equations. 1. Fundamental aspects," Journal of Physical Chemistry A, Vol. 110, No. 16, 2006, pp. 5235-5256.

${ }^{6}$ Davis, M. J., "Low-dimensional manifolds in reaction-diffusion equations. 2. Numerical analysis and method development," Journal of Physical Chemistry A, Vol. 110, No. 16, 2006, pp. 5257-5272.

${ }^{7}$ Bykov, V. and Maas, U., "The extension of the ILDM concept to reaction-diffusion models," Combustion Theory and Modeling, Vol. 11, No. 6, 2007, pp. 839-862. 786.

${ }^{8}$ Lam, S. H., "Reduced chemistry-diffusion coupling," Combustion Science and Technology, Vol. 179, No. 4, 2007, pp. 767-

${ }^{9}$ Adrover, A., Creta, F., Giona, M., and Valorani, M., "Stretching-based diagnostics and reduction of chemical kinetic models with diffusion," Journal of Computational Physics, Vol. 225, No. 2, 2007, pp. 1442-1471.

${ }^{10}$ Goussis, D. A., Valorani M., Creta, F., and Najm, H. N., "Reactive and reactive-diffusive time scales in stiff reactiondiffusion systems," Progress in Computational Fluid Dynamics, Vol. 5, No. 6, 2005, pp. 316-326.
} 
${ }^{11}$ Davis, M. J. and Skodje, R. T., "Geometric investigation of low-dimensional manifolds in systems approaching equilibrium," Journal of Chemical Physics, Vol. 111, No. 3, 1999, pp. 859-874.

${ }^{12}$ Al-Khateeb, A. N., Powers, J. M., Paolucci, S., Sommese, A. J., Diller, J. A., Hauenstein, J. D., and Mengers, J. D., "One-dimensional manifolds for spatially homogeneous systems," Journal of Chemical Physics, Vol. 131, No. 2, 2009 , pp. 024118.

${ }^{13} \mathrm{Lam}, \mathrm{S} . \mathrm{H}$. and Bellan, J., "On de-coupling of Shvab-Zel'dovich variables in the presence of diffusion," Combustion and Flame, Vol. 132, No. 4, 2003, pp. 691-696.

${ }^{14}$ Robinson, J., Infinite-Dimensional Dynamical Systems: From Basic Concepts to Actual Calculations, Cambridge University Press, Cambridge, U.K., 2001, pp. 197-198, 371.

${ }^{15}$ Kee, R. J., Rupley, F. M., and Miller, J. A., Sandia National Laboratories Report No. SAND878215B, 1992.

${ }^{16}$ Sommese A. J., and Wampler C. W., The Numerical Solution of Systems of Polynomials Arising in Engineering and Science, World Scientific, Hackensack, NJ, 2005.

${ }^{17}$ Bates, D. J., Hauenstein, J. D., Sommese A. J., and Wampler C. W., BERTINI, software for numerical algebraic geometry, available at http://www.nd.edu/ sommese/bertini.

${ }^{18}$ Kee, R. J., Dixon, L. G., Warnatz, J., Coltrin, M. E., and Miller, J. A., "A Fortran computer code package for the evaluation of gas-phase multicomponent transport properties," Report No. SAND86-8246, Sandia National Laboratories, 1991. 